\title{
Preparation and Characterization of Poly Dimethylsiloxane Based on Polyurethane Hydrogels with Interpenetrating Network Structure for Contact Lenses Uses
}

\author{
Fahima Helaly ${ }^{1}$, Sanaa El- Sawy ${ }^{1}$, Ahmad Hashem ${ }^{2}$, Reda Mourad ${ }^{1 *}$ \\ ${ }^{1}$ Department of Polymers and Pigments, Division of Chemical Industries, National \\ Research Centre, Dokki, Cairo, Egypt. \\ ${ }^{2}$ Department of Chemistry, Faculty of Science, Ain Shams University, Abbasiya, \\ Cairo, Egypt.
}

\begin{abstract}
$\mathbf{T}$ HE INTERPENETRATING polymer network (IPN) silicone hydrogels have been synthesized using polydimethylsiloxane-polyurethane- polyethyleneglycol methacrylate (PDMS-PU-PEGMA) prepolymer with different concentrations of $N, N$ dimethyl acrylamide (DMA), polyethyleneglycol methacrylate (PEGMA) and methacryloxypropyltris (trimethylsoloxy) silane (TRIS) by UV initiated polymerization process in the presence of free radical photoinitiator. The physicomechanical and chemical properties of the IPN silicone hydrogels, as water content, protein resistance, contact angle, optical transparency and mechanical properties were evaluated. The results revealed that DMA and PEGMA incorporated in IPN silicone hydrogel improves the hydrophilicity of silicone hydrogel with higher optical transparency and better protein resistance ability. The moduli of IPN silicone hydrogels indicate stiffer network as the concentration of PDMS-PU-PEGMA prepolymer increased. IPN silicone hydrogels belong to hydrophilic surfaces with the lowest water contact angle of $48.76^{\circ}$.
\end{abstract}

\section{Introduction}

Polydimethylsiloxanes (PDMS) are extensively applied in different types of industrial uses and consumer goods since their commercial introduction in 1943[1]. These substances are widely applicable in popular consumer products such as cosmetics, textiles, medicinal implants, pharmaceuticals and in industry (lubricants, coatings, gels, adhesives, antifoaming agent) [2-4]. This is attributed to their high oxygen transmissibility[5], but the earlier attempts of contact lenses production made from this material failed because silicone has hydrophobic property. Different techniques have been employed as an attempt to improve these lens properties, such as plasma- surface treatment for increasing its hydrophilicity, and creating significant edge lift for better tear exchange and diminished suction. The hydrogel component provides the comfort with suitable mechanical properties, and the silicone component provides the required oxygen performance. Usually, silicone hydrogels can be synthesized through either copolymerization or interpenetrating polymer network (IPN) technique.

Interpenetrating polymer networks are described as polymer blends containing two or more networks which are at least partially interweave on a molecular scale but there is no chemical bonds between each other and cannot be insulated unless chemical bonds are broken[6]. They are sort of elastomers in which two chemically separate networks are coexisting in a homogeneous structure to the segmental level[7]. The two networks are present as co- compatible, overlapping networks. This compatibility can be accomplished by kinetic retention of an initially miscible monomer mixture used to form the chains of network, with inhibition of phase separation by the network structure, or the result of the constituent polymers compatibility thermodynamic. It can comprise the properties of 
several polymeric materials into a new material with the mechanical properties enhancement [8]. A particular type of IPN depends on the solvent used to advance the two polymers miscibility[9]. Silicone hydrogels can be produced by either sequential IPN or simultaneous IPN. Silicone hydrogels with sequential IPN involves polymerization of hydrophilic network in the presence of swollen siloxane rubber. Silicone hydrogels with simultaneous IPN comprise polymerization of both networks concurrently at a similar rate by distinct and non meddling routes. IPN techniques dominate thermodynamic incompatibility and give materials with small phase separation and high optical clarity because of permanent overlapping networks[10]. Silicone hydrogels with IPNs have been produced by phase separation.

Wang and $\mathrm{Li}$ [11], produced a silicone hydrogel based on simultaneous IPN using hybrid freeradical/ cationic photopolymerization. The resulting IPN silicone hydrogels have hydrophilic surfaces and excellent oxygen permeability. Sequential technique may be out of shape for silicone hydrogels synthesis during the preparation process. The main characteristic of simultaneous technique over sequential technique appears in the accommodation, high efficiency and economical suitability[10-12].

The difficult incompatibility of PDMS components and hydrophilic monomers resulting in opaque, phase segregated products has been discussed by patents. This can be resolved by the modification of PDMS components to macromers with polar or hydrophilic side-chains. Such macromers are supposed to possess improved compatibility with hydrophilic monomers[13]. Moreover, the polysiloxane-polyurethane rubbers can be polymerized in form of IPN with vinyl polymer by free-radical polymerization; these IPN's are often transparent and highly oxygen permeable, permitting the physical properties of the polysiloxane-polyurethane rubber to be modified over a wide range[14].

The polysiloxane-polyether block polyurethane acrylates would show incredible photo-polymerization efficiency and low cost, owing to the high degree of functionality and the incorporated characteristics of polysiloxane fragment and polyethylene glycol fragments, without need to other excellent performances[15].

The goal of this study was improving the hydrophilicity and biocompatibility of PDMS-PU-PEGMA prepolymer through the synthesis of simultaneous IPN silicone hydrogel network by UV-photopolymerization process of acrylate monomers including PDMS-PUPEGMA prepolymer, methacryloxypropyltris (trimethylsiloxy) silane (TRIS) and N,Ndimethylacrylamide (DMA)/N-vinyl-2pyrrolidone (NVP)/polyethylene glycol methacrylate (PEGMA) in the presence of free radical photoinitiator. Also, the physicomechanical and chemical properties of the investigated simultaneous IPN silicone hydrogels were evaluated.

\section{Experimental}

Materials and methods

Poly(dimethylsiloxane) dialkanolmacromer having hydroxyethylpropoxy end groups (HO-PDMS-OH, 30-50 cSt, MW= 1000) and 3-Methacryloxypropyl tris(trimethylsoloxy) silane (TRIS) (98\%) were purchased from Gelest Co. (Morrisville, PA, USA). $N, N$ Dimethyl acrylamide (DMA), ethylene glycol dimethacrylate (EGDMA), dibutyltin dilaurate (DBTDL), sodium dodecyl sulfate (SDS) and $\mathrm{N}$-vinyl pyrrolidone (NVP) were purchased from Merck Co. (Hohenbrunn, Germany). Poly(ethylene glycol) methacrylate (PEGMA, $\mathrm{Mn}=360$ ), isophoronediisocyanate (IPDI) and Photoinitiator Darocur 1173 (2-hydroxy-2-methyl propiophenone) were purchased respectively from Sigma Co. (Louis, MO, USA).

\section{UV curable Polydimethylsiloxane- polyurethane (PDMS-PU) prepolymer preparation}

PDMS-PU prepolymer was synthesized by two steps reaction. In the first step, IPDI and $\mathrm{P}$ HO-PDMS-OH were added into the reactor with molar ratio $(2: 1)$ in the presence of catalyst DBTDL at $80-85^{\circ} \mathrm{C}$ under nitrogen gas. After about $24 \mathrm{hr}$ reaction time, PEGMA and tetrahydrofuran (THF) were added to the system as a chain extender and a solvent. Chain extension of the prepolymer was performed for $6 \mathrm{hr}$ at $80^{\circ} \mathrm{C}$. Finally, the mixture was covered with aluminum foil and stirred overnight at room temperature.

Preparation of interpenetrating polymer network (IPN) silicone hydrogel by simultaneous interpenetrating polymer network technique

Formulations of the simultaneous inter penetrating polymer network(IPN) silicone hydrogels are shown in Table1, in which PDMSPU prepolymer, TRIS, DMA and PEGMA were

Egypt. J. Chem. 61, No.1 (2018) 
used as monomers, with EGDMA as cross-linker and Darocur 1173 as photoinitiator. The mixture of each formulation was injected between two glass plates separated by silicone composite and cured under UV lamp at $365 \mathrm{~nm}$ for $1 \mathrm{hr}$. Finally, the prepared silicone hydrogel membranes were washed with $30 \%$ ethanol to remove uncured components and dried in air.

\section{Characterization}

Fourier transform infrared spectroscopy

Fourier Transform Infrared spectroscopy (FTIR) is a technique, which is used to obtain an infrared spectrum of absorption, emission or photoconductivity of a solid, liquid or gas. FTIR is based on the theory that each chemical group has characterized absorption infrared spectrum. FTIR was utilized to study the chemical structure of hydrogel and polymers, interaction of nanoparticles and hydrogel materials. The samples were scanned by FTIR in the range of $600-4000 \mathrm{~cm}^{-1}$.

\section{Equilibrium water content (EWC\%) measurement}

EWC values were obtained by immersing a piece of dry silicone samples (of previously measured mass) in a large excess of distilled water at ambient temperature for $24 \mathrm{hr}$. After removing the free water on the surface of the sample using filter paper, the mass was again measured.

$$
\mathrm{EWC} \%=\frac{(\mathrm{Wi}-\mathrm{Wd})}{\mathrm{Wi}} \times 100
$$

Where Wi and Wd are the masses of the immersed and dry samples respectively.

\section{Mechanical property measurements}

A hydrated sample of the prepared silicone hydrogel membranes $(2 \times 0.4 \mathrm{~cm})$ was mounted in a Zwick Roell test system. The samples were stretched uniaxial with a load range of $100 \mathrm{~N}$ applied on the tensile test and a crosshead speed of $5 \mathrm{~mm} / \mathrm{min}$ at room temperature. The Young's modulus (E), tensile strength and elongation at break were measured.

\section{Transparency measurement (Light Transmittance)}

The optical transparency was conducted by measuring the percent transmittance of visible light (wavelength range from 450 to $750 \mathrm{~nm}$ ) through swollen silicone samples in distilled water using a UV/Vis spectrophotometer (Agilent Technologies, Cary series UV-Vis spectrophotometer). The samples were placed between two glass slides (about $0.5 \mathrm{~mm}$ thick) and transparency was measured at $25^{\circ} \mathrm{C}$.

\section{Contact angle measurements}

Contact angle was visually measured using digital camera (Sony, DCR-SX22E) at room temperature with $1 \mu \mathrm{l}$ distilled water using micropipette. The results based on at least five surface spots.

\section{Protein adsorption assay}

Protein adsorption of artificial implants leads to protein fouling, which causes inflammatory response to human body, therefore the protein adsorption of hydrogels is another important index. The samples were immersed in PBS (phosphate buffer solution) for $24 \mathrm{hr}$, and then soaked in $0.5 \mathrm{mg} / \mathrm{ml}$ bovine serum albumin (BSA) in PBS solution for $3 \mathrm{hrs}$ at $37^{\circ} \mathrm{C}$. The silicone hydrogel samples were removed from the BSA-PBS solution and immersed into $10 \mathrm{ml}$ of PBS (3times $\mathrm{X} 10 \mathrm{mins}$ each) to remove the non-absorbed BSA on the surface of hydrogel,then blotted on filter paper, and placed into $3 \mathrm{ml}$ of $1 \mathrm{wt} \%$ SDSPBS solution for $16 \mathrm{hr}$ at room temperature to completely detach BSA from hydrogel surface to the solution. Finally, the bicinchoninic acid (BCA) protein assay kit (Micro BCA Protein Assay Kit, Boster Biological Technology Co., Valley Ave, Pleasanton, CA, USA) was used to determine the protein concentration in SDS-PBS solution with a UV-Vis plate reader at the wavelength of $562 \mathrm{~nm}$.

\section{$\underline{\text { Results and Discussion }}$}

Polydimethylsiloxane-based UV-curable polyurethane PDMS-PU prepolymer was synthesized as shown in Scheme Fig. 1.

The IPN silicone hydrogel of ultravioletcurable polyurethane (PDMS-PU) prepolymer and different concentrations of hydrophilic monomers (DMA and PEGMA) were formed according to Scheme Fig. 2 with good homogeneity as shown in Fig. 3. The replacement effect of TRIS content of polyurethane was studied.

The functional groups of IPN silicone hydrogels were characterized by FTIR. Their equilibrium water content, mechanical properties, protein adsorption and water contact angle were also measured.

\section{FTIR analysis}

FTIR spectra for the poly (dimethylsiloxane) dialkanol macromer (HO-PDMS-OH) and PDMSPU prepolymer, shown in Fig. 4, confirmed the formation of PDMS-PU prepolymer. The spectra of PDMS-PU prepolymer showed characteristic peaks at $3338 \mathrm{~cm}^{-1}$ (NH stretching), $2901 \mathrm{~cm}^{-1}$ and $2957 \mathrm{~cm}^{-1}$ 
$\left(-\mathrm{CH}_{3}\right.$ and $\left.-\mathrm{CH}_{2}\right), 1718 \mathrm{~cm}^{-1}(\mathrm{C}=\mathrm{O}$ stretching $)$, $1642 \mathrm{~cm}^{-1}(\mathrm{C}=\mathrm{O}$ urethane group, $\mathrm{C}=\mathrm{C}$ stretching $)$, $1456 \mathrm{~cm}^{-1}$ (C-N stretching), and $1256 \mathrm{~cm}^{-1}$ (Si$\mathrm{CH}_{3}$ stretching), $1025 \mathrm{~cm}^{-1}$ (Si-O stretching). The spectra also indicated the disappearance of peak at $2260 \mathrm{~cm}^{-1}$ which is chrachteristic peak for the isocyanate group $(\mathrm{N}-\mathrm{C}=\mathrm{O})$. This means that the amount of isophorondiisocyante was consumed and the reaction was completed.

FTIR spectra of the IPN silicone hydrogels derived from formulations R600, R602 and R332 as cited in Table 1 are shown in Fig.5. The spectrum showed peaks at $1720 \mathrm{~cm}^{-1}$ and $1638 \mathrm{~cm}^{-1}$ characteristics for $\mathrm{C}=\mathrm{O}$ group and its intensity at $1720 \mathrm{~cm}^{-1}$ increased with the content of acrylate monomers (PEGMA, and TRIS). Peaks at $1260 \mathrm{~cm}^{-1}$ and $1037 \mathrm{~cm}^{-1}$ are due to $\mathrm{Si}-\mathrm{CH}_{3}$ and $\mathrm{Si}-\mathrm{O}$ stretching groups. The peaks at $3430 \mathrm{~cm}^{-1}$ are characteristic of $\mathrm{N}-\mathrm{H}$ stretching in case of formulation $\mathrm{R} 600$ and for $\mathrm{N}-\mathrm{H}$ and $\mathrm{OH}$ stretching in case of formulations R602 and R332 where their intensities increased.

\section{Equilibrium water content (EWC \%)}

The equilibrium water content of IPN silicone hydrogels is shown in Table 2 and Fig. 6. It is clear that, the equilibrium water content decreased as the amount of DMA decreased and the amounts of both PEGMA and TRIS increased. It is found that, when PDMS- PU prepolymer was used without TRIS replacement, the hydrophilicity of IPN silicone hydrogels increased and the EWC reached the highest values of $41.5 \%$ and $48 \%$ when the ratios of PDMS- PU: DMA were 60:40 and 50:50 for formulations R600 and R500 respectively. Fig. 10 also revealed that when PEGMA replaced parts of DMA, the EWC was decreased slightly to $38.9,37,46.3$ and $44.7 \%$ when the ratio of PDMSPU:DMA:PEGMA was 60:30:10, 60:20:20, 50:40:10 and 50: 30:20 formulations R601, $\mathrm{R} 602, \mathrm{R} 501$ and R502 respectively. It is observed that when TRIS content increased and PDMS-PU prepolymer decreased, the EWC dropped slightly.
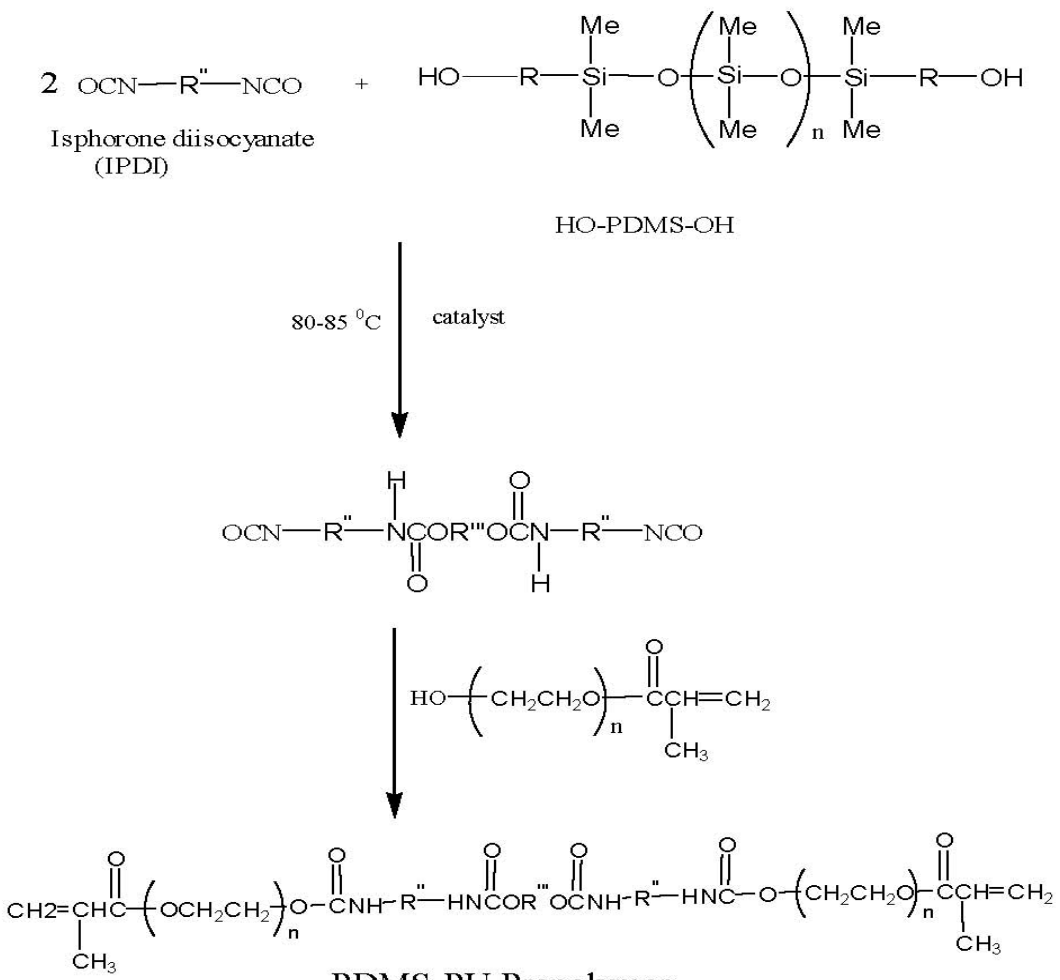

PDMS-PU Prepolymer

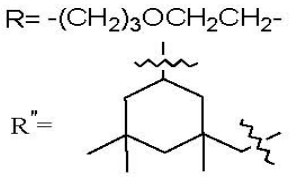

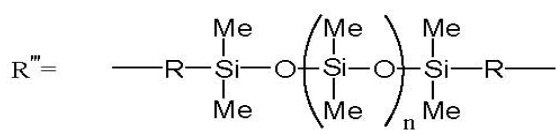

Fig.1. Chemical scheme of UV-curable PDMS-PU prepolymer synthesis. 
When the ratios of PDMS-PU:TRIS were $60 \%$ (50:10, 40:20 and 30:30) and 50\% (40:10, 30:20 and 20:30), the EWC recorded $36.8,34.5,34.4$ and $44.3,44.1,43.6 \%$ at $10 \%$ PEGMA and at $20 \%$ PEGMA 35.5, 34.5, 32 and 42.3, 42.2, 41.7\% respectively.

The highest equilibrium water content values can be attributed to the presence of DMA which specified with high hydrophilicity and acquires much higher water content to a hydrogel than alcohol-containing monomers. Also, it copolymerizes well with methacrylates[16]. The permeation of water through silicone hydrogels lenses was suitable when possessing enough wettability, comfort, and on-eye lens movement [10]. This starts when the water content in the lens reaches about 20\% [17].

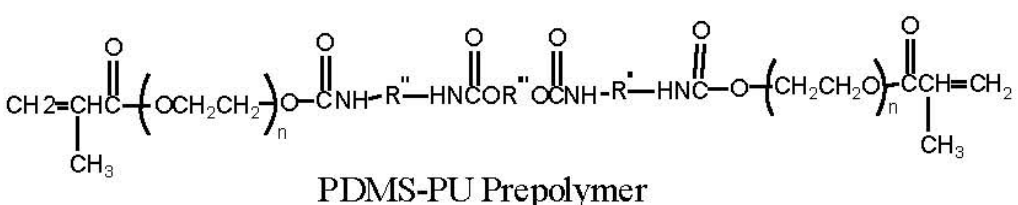

PDMS-PU Prepolymer<smiles>C=C(C)C(=O)OCCC[Si](O[Si](C)(C)C)(O[Si](C)(C)C)O[Si](C)(C)C</smiles><smiles>C=CC(=O)N(C)C</smiles>

DMA<smiles>C=C(C)C(=O)C(C)CCC(C)(C)O</smiles>

PEGMA

UV-photopolymerization

\section{IPN Silicone hydrogel}

Fig . 2. Chemical scheme of simultaneous IPN silicone hydrogel formation. The TRIS, DMA, PEGMA contents of IPN were varied by changing their weight percent during synthesis. 

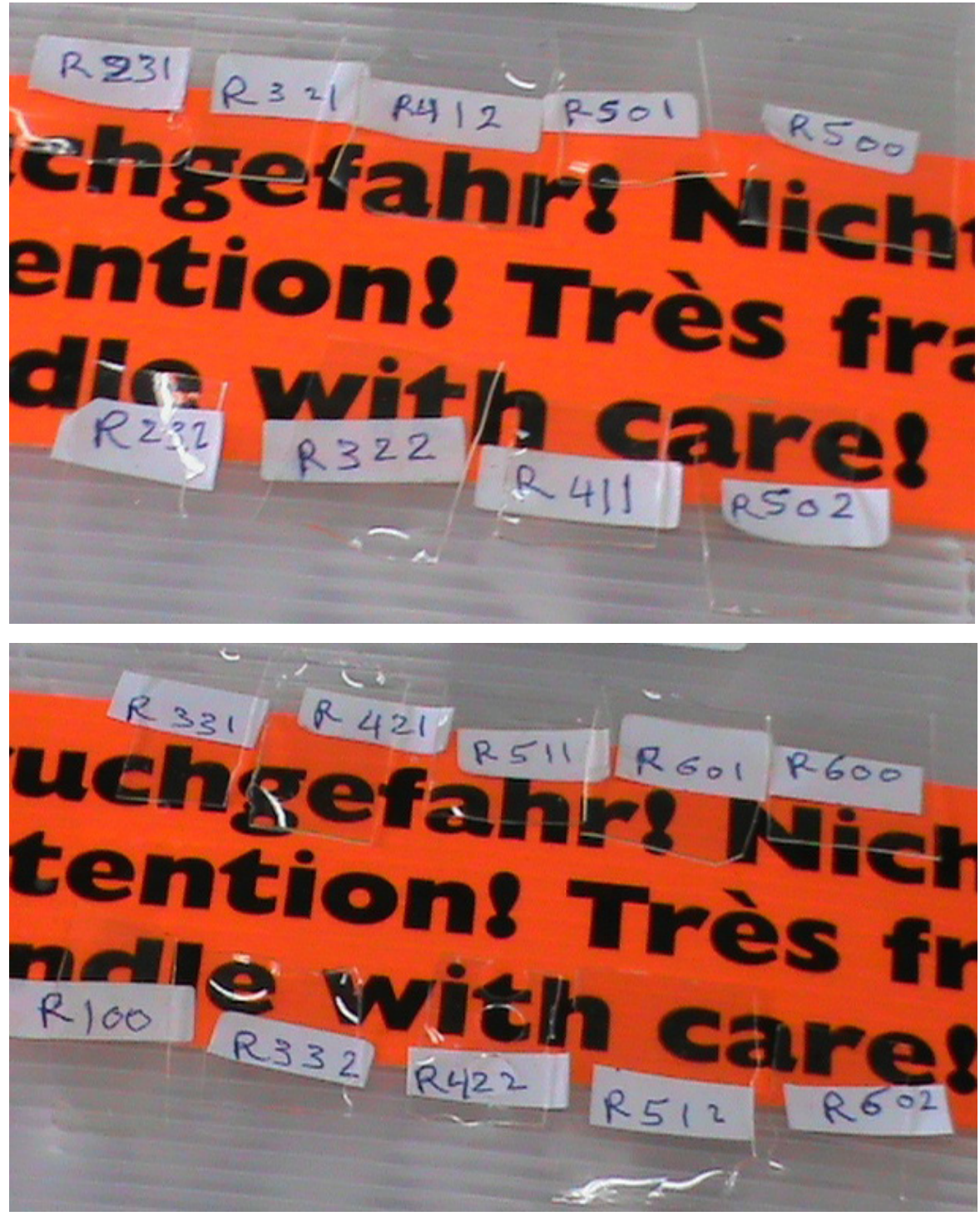

Fig. 3. Digital photo of IPN silicone hydrogel membranes.

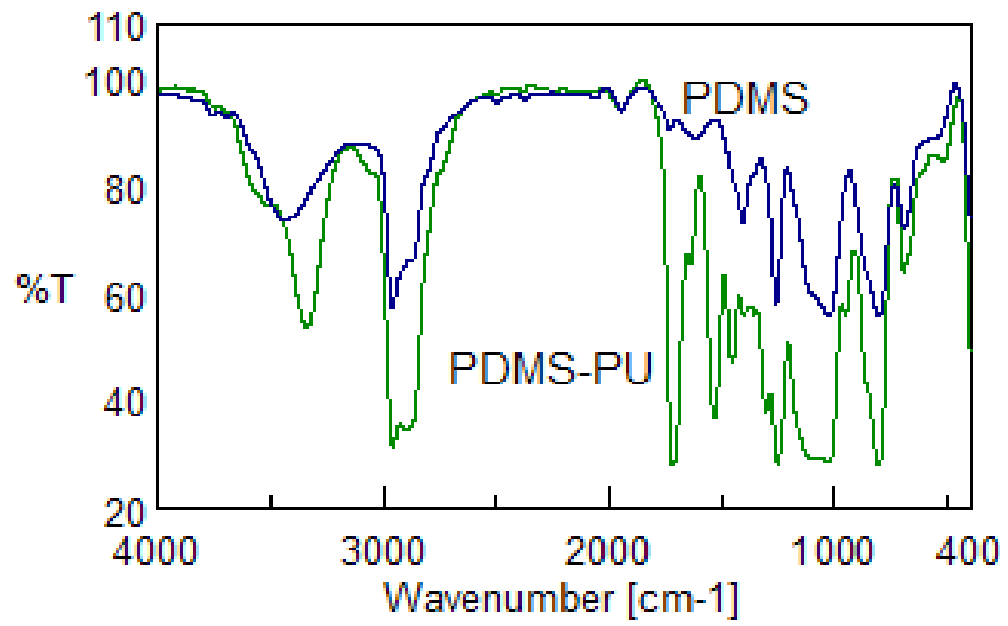

Fig. 4. FTIR spectra of poly(dimethylsiloxane) dialkanolmacromer (HO-PDMS-OH) and PDMS-PU prepolymer. 


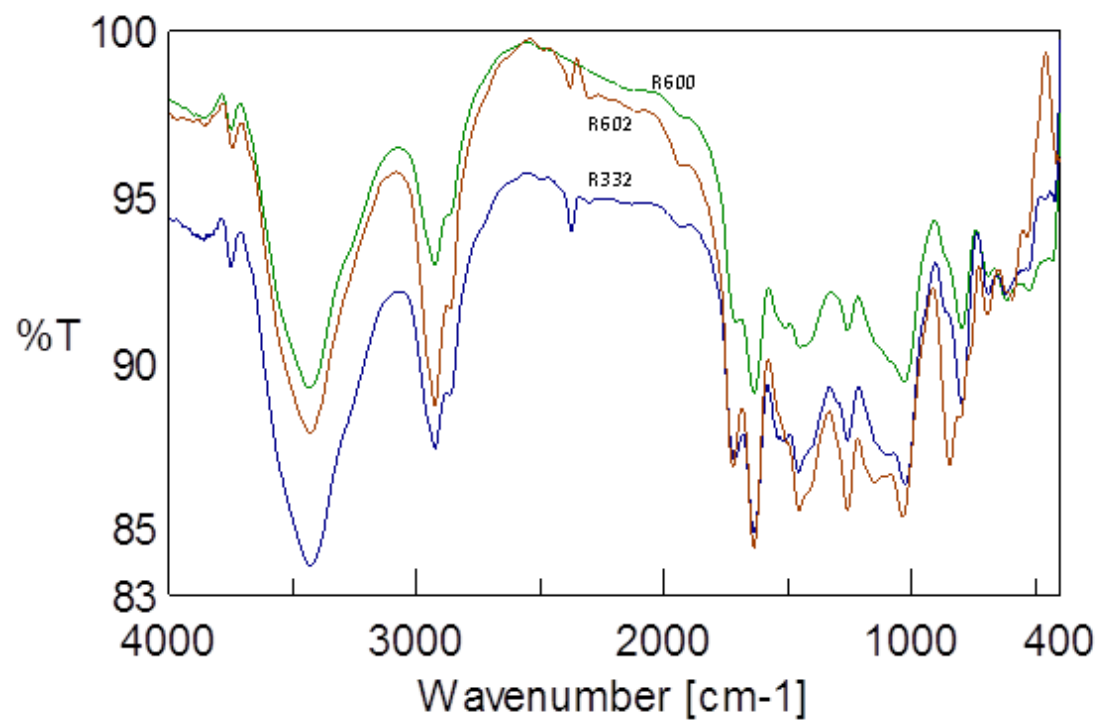

Fig. 5. FTIR spectra of simultaneous IPN silicone hydrogels derived from: formulations (R600, R602 and R332) as cited in Table 1.

TABLE 1. Formulations of simultaneous IPN silicone hydrogels.

\begin{tabular}{|c|c|c|c|c|c|}
\hline Formulation No & $\begin{array}{c}\text { PDMS-PU: TRIS } \\
\text { wt (\%) }\end{array}$ & $\begin{array}{c}\text { DMA } \\
\text { wt (\%) }\end{array}$ & $\begin{array}{l}\text { PEGMA } \\
\text { wt (\%) }\end{array}$ & $\begin{array}{l}\text { EGDMA } \\
\text { wt (\%) }\end{array}$ & $\begin{array}{c}\text { Darocur } 1173 \\
\text { wt }(\%)\end{array}$ \\
\hline R100 & $100: 0$ & 0 & 0 & 0 & 1 \\
\hline R600 & 60: 0 & 40 & 0 & 0.3 & 1 \\
\hline R601 & $60: 0$ & 30 & 10 & 0.3 & 1 \\
\hline R511 & 50: 10 & 30 & 10 & 0.3 & 1 \\
\hline R421 & 40: 20 & 30 & 10 & 0.3 & 1 \\
\hline R331 & $30: 30$ & 30 & 10 & 0.3 & 1 \\
\hline R500 & 50: 0 & 50 & 0 & 0.3 & 1 \\
\hline R501 & 50: 0 & 40 & 10 & 0.3 & 1 \\
\hline R411 & 40: 10 & 40 & 10 & 0.3 & 1 \\
\hline R321 & $30: 20$ & 40 & 10 & 0.3 & 1 \\
\hline $\mathrm{R} 231$ & 20: 30 & 40 & 10 & 0.3 & 1 \\
\hline R602 & 60: 0 & 20 & 20 & 0.3 & 1 \\
\hline R512 & 50: 10 & 20 & 20 & 0.3 & 1 \\
\hline R422 & 40: 20 & 20 & 20 & 0.3 & 1 \\
\hline R332 & 30: 30 & 20 & 20 & 0.3 & 1 \\
\hline R502 & 50: 0 & 30 & 20 & 0.3 & 1 \\
\hline R412 & 40: 10 & 30 & 20 & 0.3 & 1 \\
\hline R322 & 30: 20 & 30 & 20 & 0.3 & 1 \\
\hline R232 & $20: 30$ & 30 & 20 & 0.3 & 1 \\
\hline
\end{tabular}


TABLE 2. The equilibrium water content (EWC\%) and contact angle of IPN silicone hydrogels.

\begin{tabular}{|c|c|c|}
\hline Formulation No & EWC, \% & $\begin{array}{c}\text { Contact angle, } \\
\text { Degree }\end{array}$ \\
\hline R100 & 6.53 & 103.5 \\
\hline R600 & 41.57 & 56.32 \\
\hline R601 & 38.9 & 58.62 \\
\hline R602 & 37.05 & 60.27 \\
\hline R511 & 36.85 & 71.51 \\
\hline R512 & 35.48 & 74.65 \\
\hline R421 & 34.47 & 77.54 \\
\hline R422 & 34.45 & 78.3 \\
\hline R331 & 34.35 & 79.77 \\
\hline R332 & 32.05 & 81.02 \\
\hline R500 & 47.99 & 48.76 \\
\hline R501 & 46.33 & 51.76 \\
\hline R502 & 44.73 & 56.33 \\
\hline R411 & 44.34 & 58.04 \\
\hline R412 & 42.31 & 61.55 \\
\hline R321 & 44.13 & 59.67 \\
\hline R322 & 42.22 & 63.34 \\
\hline R231 & 43.57 & 60.47 \\
\hline R232 & 41.68 & 64.2 \\
\hline
\end{tabular}

TABLE 3. The young's modulus(E), tensile strength (T.S.) and elongation at break (E) of IPN silicone hydrogels.

\begin{tabular}{ccccc}
\hline Formulation No & TRIS\% & E, & T.S., & \&, \\
& & MPa & MPa & 20.94 \\
\hline R601 & 0 & 3.05 & 0.55 & 26.12 \\
R511 & 10 & 2.63 & 0.45 & 32.9 \\
R421 & 20 & 2.16 & 0.38 & 72.74 \\
R331 & 30 & 0.89 & 0.35 & $\%$ \\
\hline
\end{tabular}

Egypt. J. Chem. 61, No.1 (2018) 

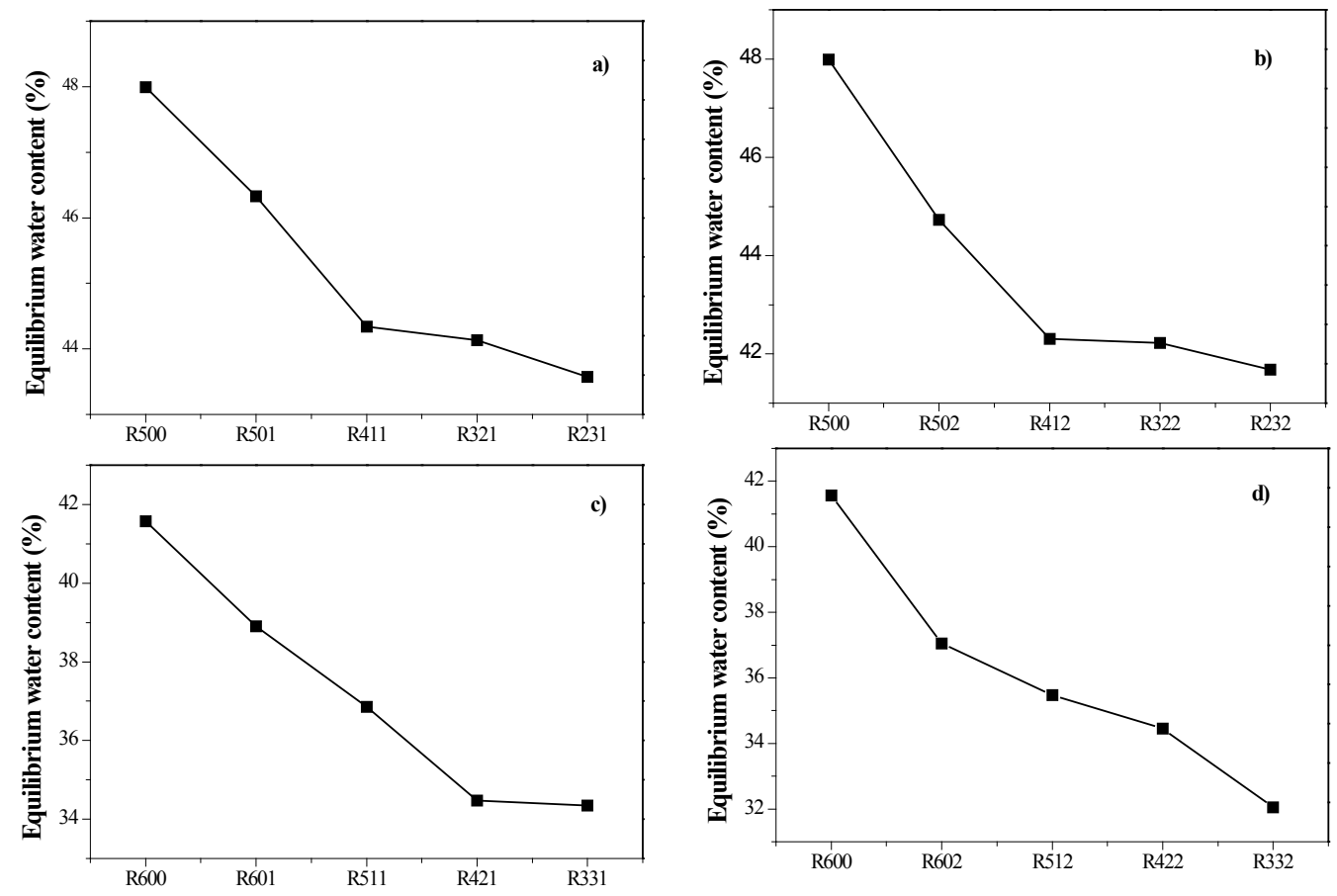

Fig. 6. Equilibrium water content of simultaneous IPN silicone hydrogels derived from the formulations as cited in Table 3.

\section{Mechanical properties}

The modulus of the IPN silicone hydrogels tested in the hydrated state are listed in Table 3 and illustrated in Fig. 7. The IPN silicone hydrogels used for the mechanical property characterization were prepared with the same content of hydrophilic monomers (30\% of DMA and $10 \%$ of PEGMA), while the combined amount of the PDMS-PU prepolymer and TRIS was $60 \%$ in different formulations. The results revealed that the modulus decreased with the increase of the TRIS content in the formulations. This could be explained that the increase of TRIS content reduced the overall crosslinking density of the hydrogels. The silicone hydrogels possessed modulus ranging from $3 \mathrm{MPa}$ to $0.89 \mathrm{MPa}$; without TRIS, formulation R601 recorded the highest modulus value of $3 \mathrm{MPa}$, but when TRIS was used to replace 10 parts of PDMS-PU prepolymer, the modulus dropped to 2.6 MPaformaltion R511; when about 20 parts replaced PDMS-PU prepolymer, it was $2.1 \mathrm{MPa}$ for formulation R421 and when 30 parts replaced PDMS-PU prepolymer it became $0.89 \mathrm{MPa}$ for formulation R331. As shown in Table 3, the increase of TRIS content increased the IPN silicone hydrogel elongation at break, while tensile strength decreased gradually. These results revealed that the IPN silicone hydrogels possessed mechanical properties as good materials for the contact lens use specially for formulation R332. So it is thought to be a promising material. The modulus of a lens has important consequences on vision correction and safety. Young's modulus of the commercial silicone hydrogel lenses is around 0.5 to $1.5 \mathrm{MPa}[18]$. Moreover, the increase in rigidity or stiffness of the IPN silicone hydrogels could acquire some advantages of lenses handling and more corneal astigmatism is masked compared to flexible hydrogels[19-20].

\section{Transparency measurements}

The transparency of the silicone hydrogels is an important parameter for the contact lens application. Fig.8 and 9 show the transparency of the IPN silicone hydrogels measured by UVVis spectrophotometer at a wavelength range between $450 \mathrm{~nm}$ and $750 \mathrm{~nm}$. It is clear that, light transmission by all prepared IPN silicone hydrogels was above $92 \%$. The transparency of the all IPN silicone hydrogels revealed the homogeneous phase structure of the two networks of hydrophilic and hydrophobic ones i.e. there is a micro-phase separation causing slight reduction in optical transparency. It has been reported that soft contact lenses should have light transmittance of above $90 \%$ [21-22]. 


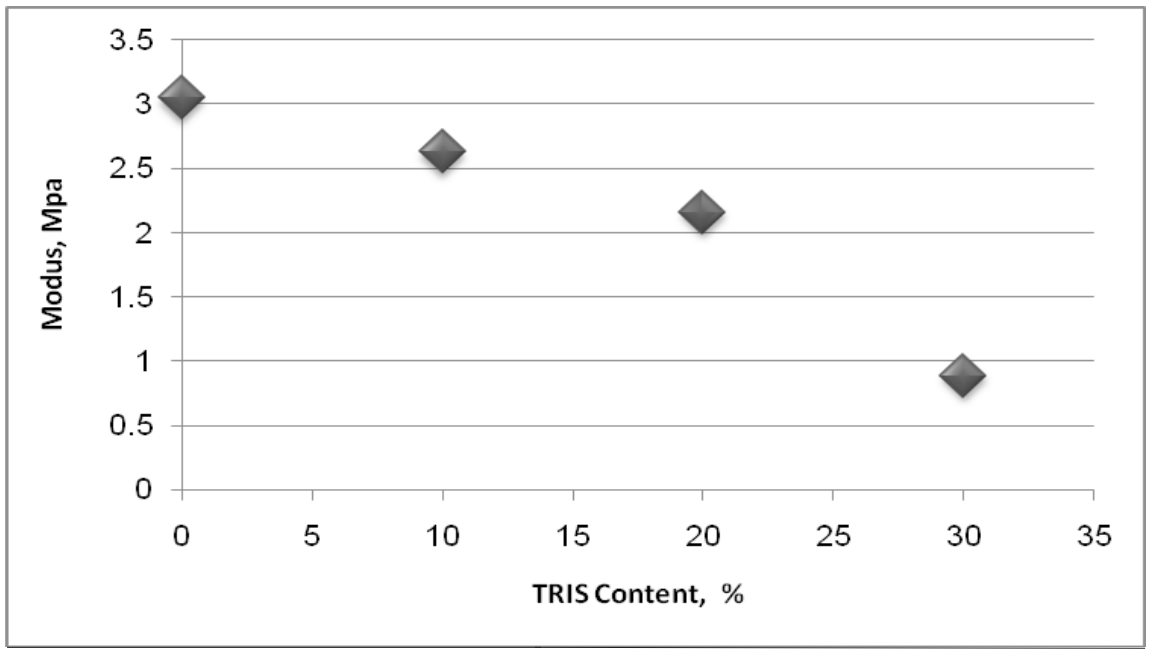

Fig. 7. The relationship between tensile modulus and TRIS content at 30\% DMA and 10\% PEGMA.
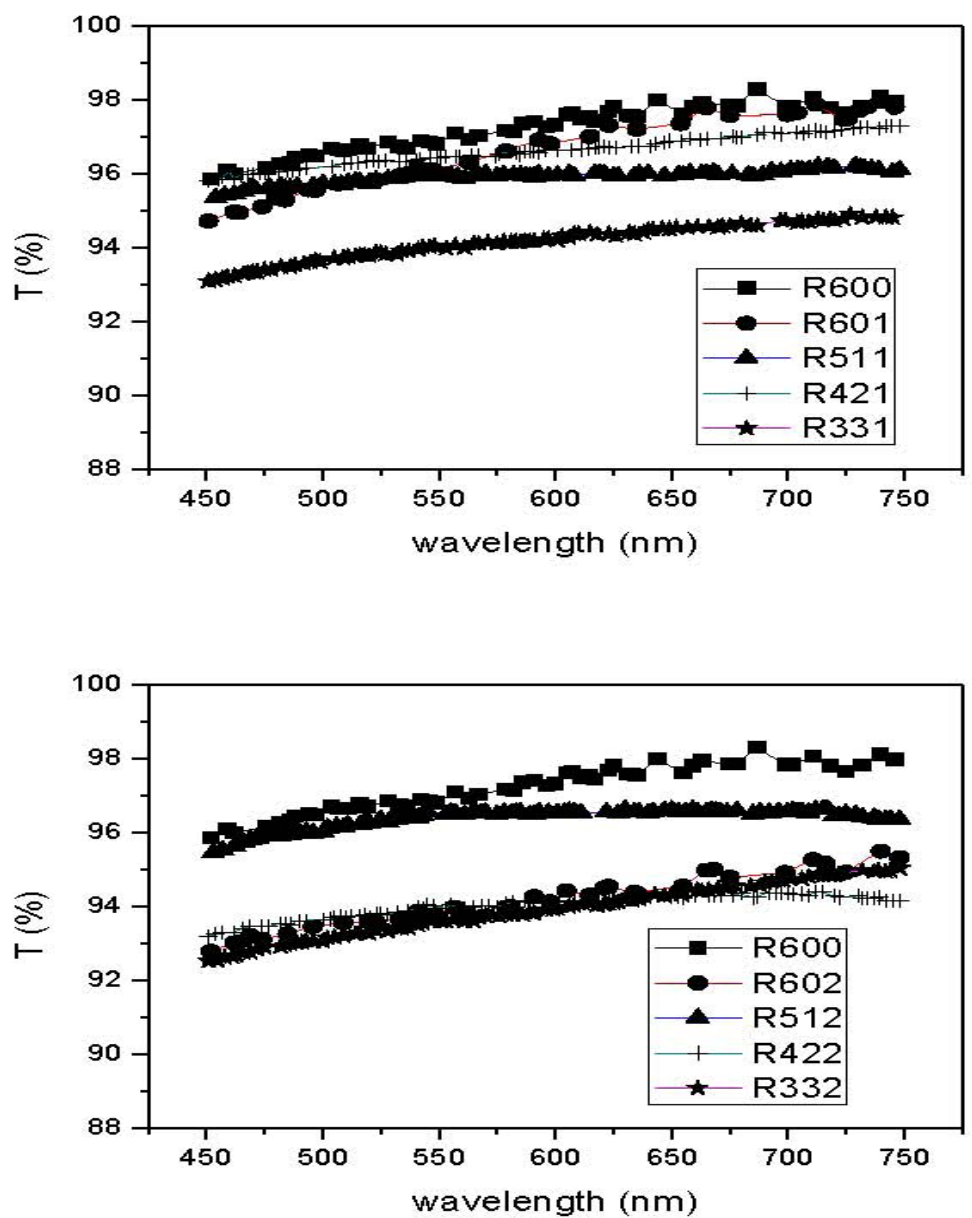

Fig. 8. Transmittance of IPN silicone hydrogels at 50\% (PDMS-PU:TRIS): 50\% (DMA:PEGMA) from the formulations as cited in Table 1.

Egypt. J. Chem. 61, No.1 (2018) 

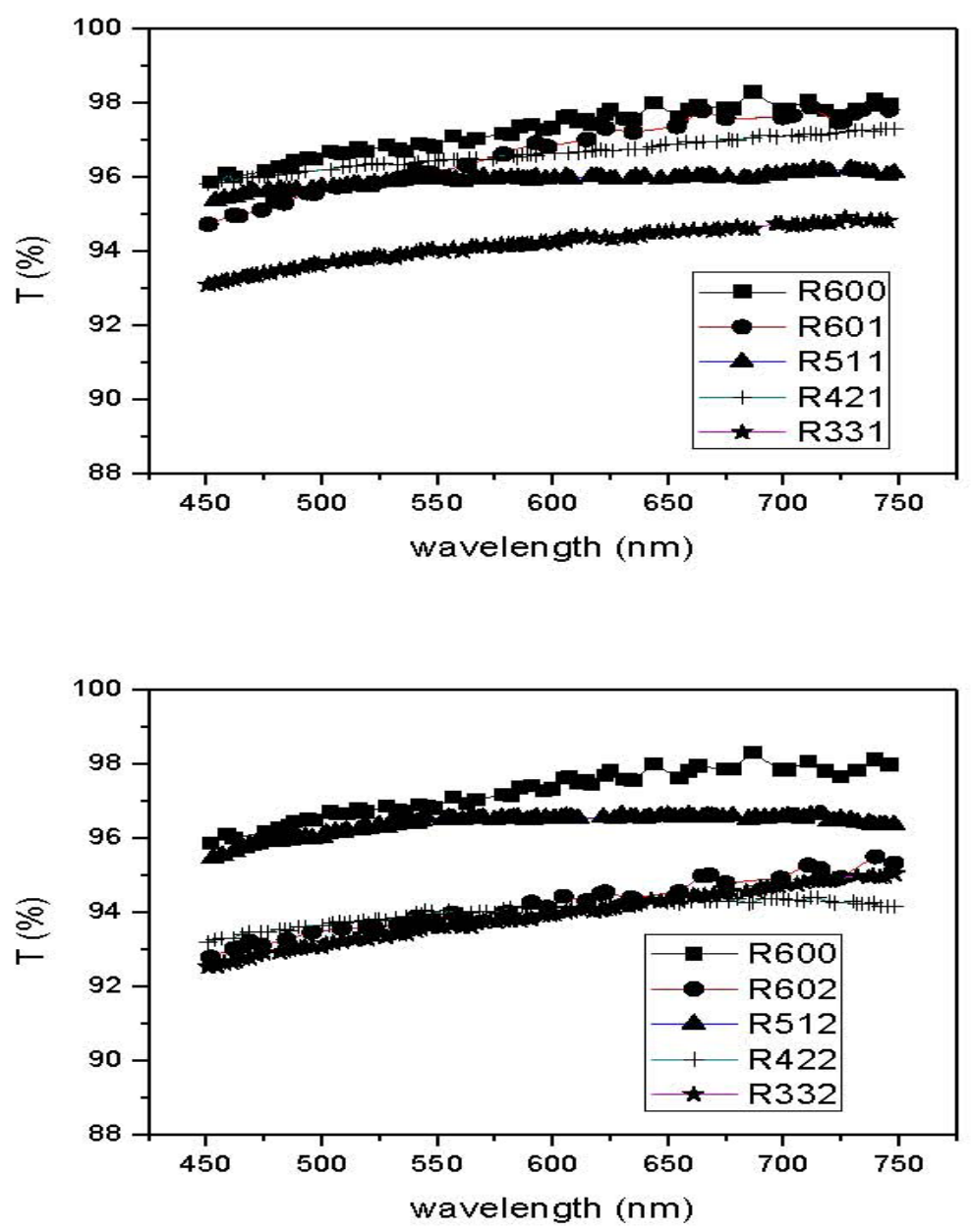

Fig. 9. Transmittance of IPN silicone hydrogels at $60 \%$ (PDMS-PU:TRIS): $40 \%$ (DMA:PEGMA) from the formulations as cited in Table 1.

\section{Contact angle measurements}

The water contact angle of the prepared silicone hydrogel films is shown in Table 2. Although the IPN silicone hydrogel showed higher water content and increased hydrophilicity with hydrophilic monomer, a less wettability surface was displayed by these IPN silicone hydrogels. It is found that, when the TRIS content increased, the water contact angle of the hydrogel increased. This can be explained that the TRIS monomer was an effective factor in the surface wettability of the resulting IPN silicone hydrogel. Additionally, since PDMS had a much lower surface energy than polyurethane, PDMS might migrate to the surface layer during the hydrogel formation, resulting in a low surface energy as well as high water contact angle. The IPN silicone hydrogel formulations R600 and R500 recorded the lowest contact angles $56.32^{\circ}$ and $48.76^{\circ}$ respectively without TRIS and PEGMA contents. The results revealed that when PEGMA replaced DMA, the contact angle increased to be $58.62^{\circ}$, $60.27^{\circ}, 51.76^{\circ}$ and $56.33^{\circ}$ formulations R601, R602, R501 and R502 respectively without TRIS content. The contact angle became continuously increased when TRIS replaced PDMS-PU prepolymerto record $71.5^{\circ}, 77.54^{\circ}, 79.77^{\circ}$ formulations R511, R421, R331 and $74.65^{\circ}, 78.3^{\circ}$, $81.02^{\circ}$ formulations R512, R422, R332 at $40 \%$ of hydrophilic part (30\% DMA:10\% PEGMA and 20\% DMA:20\% PEGMA respectively), and $60 \%$ of hydrophobic part (PDMS-PU:TRIS). Also, the contact angle gave $58^{\circ}, 59.67^{\circ}, 60.47^{\circ}$ formulations R411, R321, R231 and $61.55^{\circ}$, $63.34^{\circ}, 64.2^{\circ}$ formulations R412, R322, R232at $50 \%$ of hydrophilic part (40\%DMA:10\%PEGMA and 30\%DMA:20\%PEGMA respectively) and $50 \%$ of hydrophobic part (PDMS-PU:TRIS).

Egypt. J. Chem. 61, No.1(2018) 
Maldonado-Codina and Morgan[23], measured the water contact angles of five commercially available silicone hydrogel contact lenses (Acuvue Advance, Acuvue Oasys, Focus Night \& Day, O Optix, and PureVision) using the two most widely-used captive bubble and sessile drop (from the direct blister and after $48 \mathrm{hr}$ of soaking/ washing in saline) techniques. They found that, the water contact angle was $31^{\circ}, 66^{\circ}$ and $96^{\circ}$ (for Acuvue Advance lens), $32^{\circ}, 77^{\circ}$ and $85^{\circ}$ (for Acuvue Oasys lens), $41^{\circ}, 44^{\circ}$ and $44^{\circ}$ (for Focus Night \& Day lens), $44^{\circ}, 44^{\circ}$ and $37^{\circ}$ (for $\mathrm{O}_{2}$ Optix lens) and $30^{\circ}, 94^{\circ}$ and $102^{\circ}$ (for PureVision lens) respectively. Our results are much closer to the water contact angle of conventional silicone hydrogel lenses.
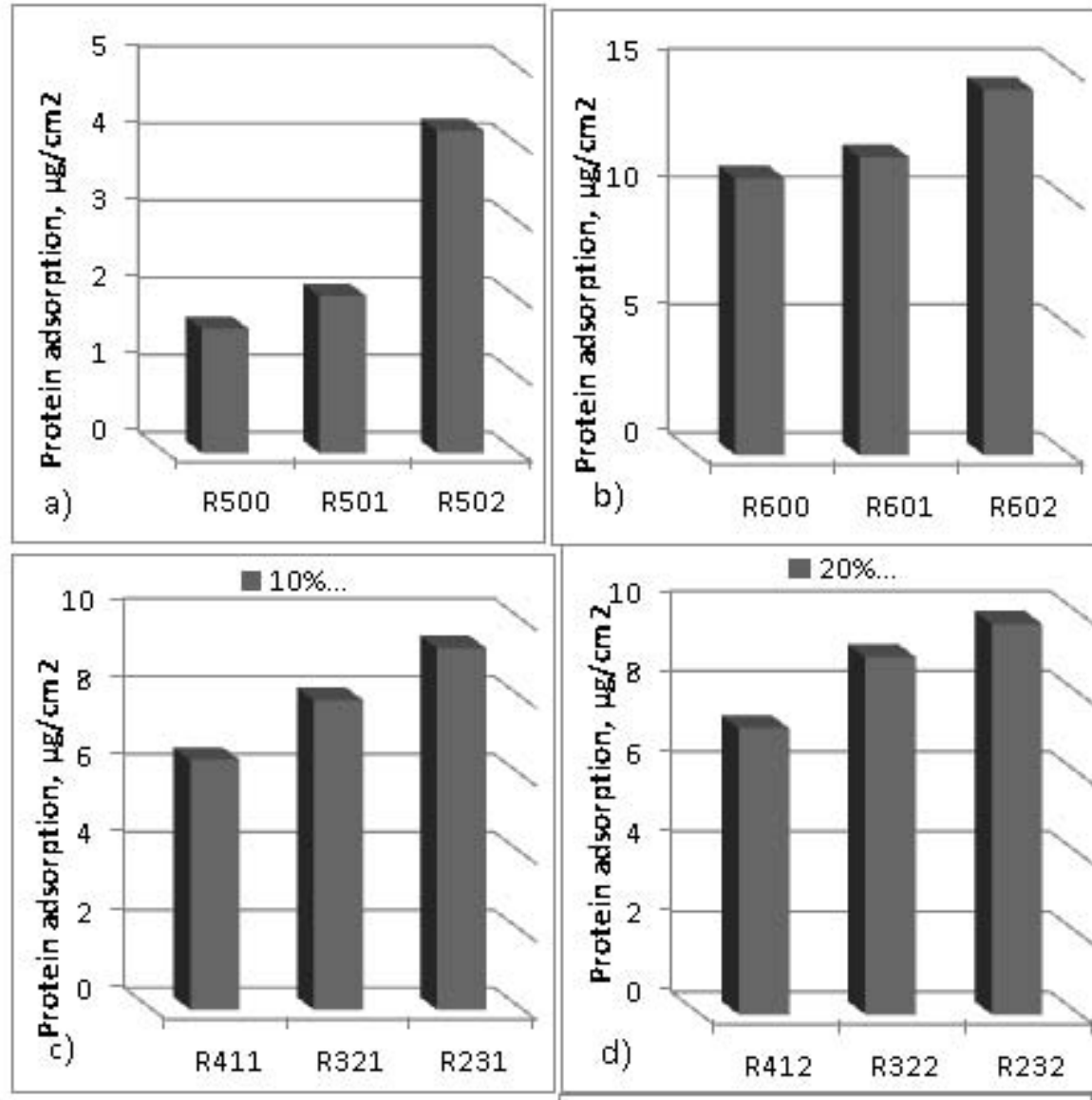

b)
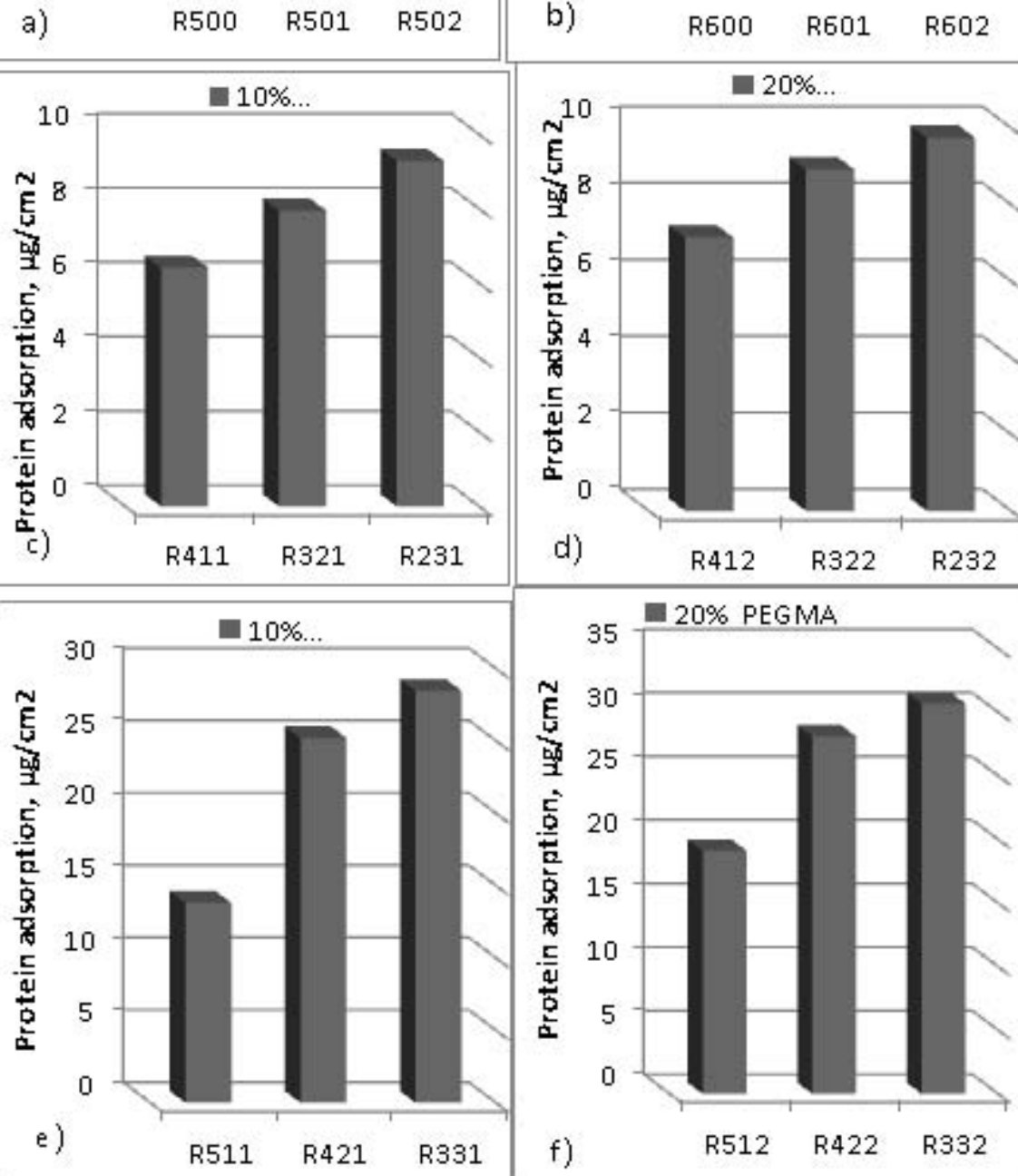

Fig.10. Bovine serum albumin (BSA) adsorption of IPN silicone hydrogels. 


\section{Protein resistance}

Protein adsorption onto the surface of the IPN silcone hydrogel was measured by the method of bicinchoninic acid assay using bovine serum albumin(BSA) as amodel. Fig.10 shows the amount of BSA adsorbed onto the IPN silicone hydrogels. As indicated in Fig.10(a,b), the amount of BSA adsorbed onto the IPN silicone hydrogels without TRIS formulations R600, R601, R602, R500, R5001 and R502 was lower than that of the IPN silicone hydrogel containing TRIS. The amount of BSA adsorptions increased from $10.82 \mu \mathrm{g} / \mathrm{cm}^{2}$ formulation R600 to $14.28 \mu \mathrm{g} / \mathrm{cm}^{2}$ formulation R602 and from $0.61 \mu \mathrm{g} / \mathrm{cm}^{2}$ formulation R500 to $4.18 \mu \mathrm{g} / \mathrm{cm}^{2}$ formulation R502 when the PEGMA content varied from $0 \%$ to $20 \%$. The amount of protein adsorption increased as the TRIS content increased. The IPN silicone hydrogels in the study showed better protein resistance ability. Usually, hydrophilic surfaces exhibit a relatively high protein resistance. The effective reduction in the protein adsorption may be attributed to the hydrophilic part comes in contact with the surrounding aqueous environment, which can prevent protein adsorption from the solution onto the surfaces. Therefore, IPN silicone hydrogels without TRIS possessed a lower affinity site for protein adsorption than IPN silicone hydrogels containing TRIS.

\section{Conclusions}

The IPN silicone hydrogels were successfully synthesized from PDMS-PU prepolymer with different concentrations of DMA, PEGMA and TRIS. The DMA and PEGMA incorporated in IPN silicone hydrogel improved the hydrophilicity of silicone hydrogel with higher optical transparency. The moduli of IPN silicone hydrogels indicated stiffer network and closer to conventional silicone hydrogel contact lens. It is worthy to state that, the protein adsorption and contact angles were in an acceptable range for use as contact lens material. Accordingly, the prepared IPN silicone hydrogel possesses characteristics that can be broadened their potential applications to ophthalmic devices as contact lenses.

\section{Acknowledgments}

We are grateful to National Research Centre for financial support through $\mathrm{PhD}$ Thesis fund of RedaMourad No. 1/3/8.

\section{References}

1. Chandra G., Maxim L. and Sawano T., The silicone industry and its environmental impact, In Organosilicon Materials. Springer, pp. 295 (1997).

2. Smith A. L., The Analytical Chemistry Of Silicones, Wiley-Interscience (1991).

3. Fendinger N., Lehmann R. and Mihaich E., Polydimethylsiloxane, In Organosilicon Materials. Springer, pp. 181 (1997).

4. Dewil R., Appels L. and Baeyens J., Energy Conversion And Management 47,1711 (2006).

5. Weissman B., Fatt I. and Pham C., Journal of the American Optometric Association 63,187 (1992).

6. Chen J., Liu M. and Chen S., Materials Chemistry and Physics 115,339 (2009).

7. Kim S. and Sperling L. H., IPNs Around The World: Science And Engineering, John Wiley \& Sons (1997).

8. Tang Q., Sun X., Li Q., Wu J. and Lin J., Colloids and Surfaces A: Physicochemical and Engineering Aspects 346,91 (2009).

9. Roland C., Encyclopedia of Polymeric Nanomaterials 1004 (2015).

10. Xu J., Zhang L., Zhang Y., Li T. and Huo G., Journal of Biomaterials Science, Polymer Edition 25,121 (2014).

11. Wang J. and Li X., Journal Of Applied Polymer Science 116, 2749 (2010).

12. Wang J. and Li X., Polymers for Advanced Technologies 22, 2091 (2011).

13. Mueller K. F., Heiber S. J. and Plankl W. L., Strong, silicone containing polymers with high oxygen permeability. U.S. Patent 4,486,577 (1984).

14. Mueller K.F. and Harisiades P., Crosslinked siloxane-urethane polymer contact lens. U.S. Patent 5, 426,158 (1995).

15. Cheng J., Cao Y., Jiang S., Gao Y., Nie J. and Sun F. , Industrial \& Engineering Chemistry Research 54, 5635 (2015).

16. Lai Y. C. and Valint P. L. , Journal Of Applied Polymer Science 61,2051 (1996).

17. N. A. Peppas and L. Brannon-Peppas, Journal of membrane science 48, 281 (1990).

18. Nicolson P. C., Baron R. C., Chabrecek P., Domschke A., Griesser H. J., Ho A. , Hopken J., Laycock B. G., Liu Q. and Lohmann D. , Extended wear ophthalmic lens. U.S. Patent 5,760,100

Egypt. J. Chem. 61, No.1(2018) 
(1998).

19. Lin C.H., Lin W.-C. and Yang M.-C., Colloids and Surfaces B: Biointerfaces 71,36 (2009).

20. Kim J., Conway A. and Chauhan A., Biomaterials 29,2259 (2008)

21. Gulsen D. and Chauhan A., International Journal of Pharmaceutics 292,95 (2005).
22. Helaly F., El-Sawy S., Hashem A., Khattab A. and Mourad R., Contact Lens and Anterior Eye 40,59 (2017).

23. Maldonado-Codina C. and Morgan P. B., Journal of Biomedical Materials Research Part A 83,496 (2007).

(Received 20/11/2017; accepted $30 / 1 / 2018$

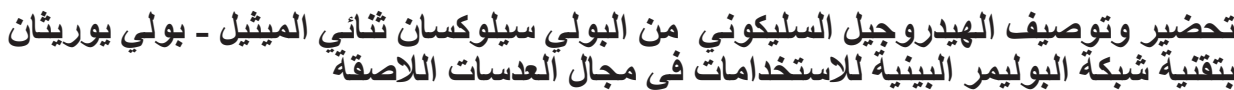

فهيمة مسعد هلالي1، سناء محمد الصاوي1، أحمد اسماعيل هاثم²، رضا محمد مراد الـات

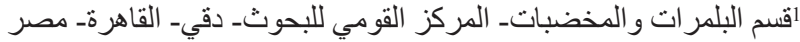

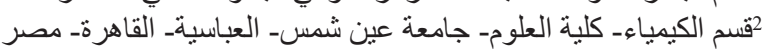

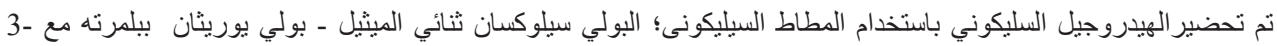

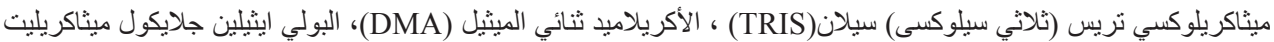

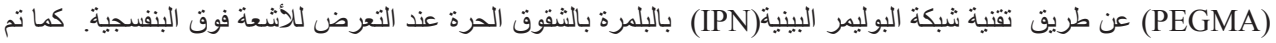

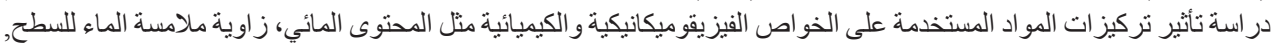

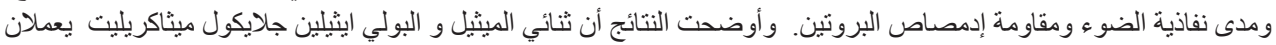

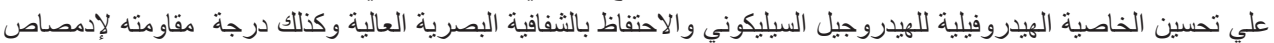

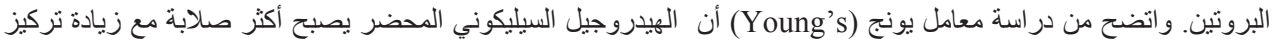

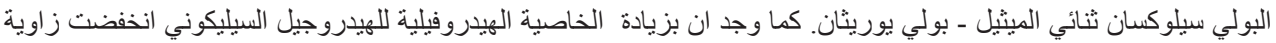

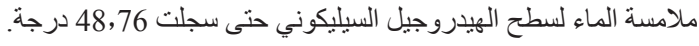

